\title{
P-0463 - Subgroup of Autoimmune Diabetes in Adults with Type 2 Diabetes
}

\author{
Anastasia Basik' ${ }^{1}$ Maxim Lushchyk², Larisa Danilova², \\ Galina Korolenko ${ }^{1}$, Elena Dashkevitch ${ }^{1}$ \\ 1 10-th city hospital, Minsk, Belarus \\ 2 Belarusian Medical Academy for Postgraduate Education, Minsk, Belarus
}

\begin{abstract}
Background. Diabetes mellitus is a heterogeneous metabolic disorder characterized by hyperglycemia. Methods of definition of etiological subgroups based on physiological features, such as insulin resistance and beta cell failure, autoimmunity are changing over time. The necessity of defining subgroups in type 2 diabetes is evident and has a major impact on the ability to optimize treatment protocol.
\end{abstract}

Aim. Our objective was the detection of autoimmune diabetes subgroup among patients with type 2 diabetes.

Methods. Adult patients with type 2 diabetes hospitalized to 10-th city hospital were invited to participate in the study. The entry criteria were - duration of type 2 diabetes for three years, therapy with oral antidiabetic drug (OAD), onset of diabetes at the age of 40-45 years and older, excessive BMI (varied from 32,2 to $38.5 \mathrm{~kg} / \mathrm{m} 2$ ); 71 patient was enrolled (28 male and 43 female) [fig.1]. General clinical characteristics of participants, history of diabetes, metabolic parameters, complications were analyzed. Pre- and postprandial insulin, C-peptide levels, GAD65 and TPO antibodies in blood serum were measured. Thyroid ultrasound was done in all patients. Thyroid function parameters were checked. The most common treatment regimen was the dual therapy with metformin and another OAD (sulphonylureas), followed by triple therapy with metformin and two additional OAD (sulphonylureas and DPP4).

Results. By the time of hospitalization all patients showed a failure to achieve target glycemic levels. Glycated hemoglobin AIc (HbAIc) ranged from 9.5 to $13 \%$. Among them 54/71 had low C-peptide serum levels (less than the lower limit of the normal range and inadequate increase after a standard breakfast load in 2 hours) [fig.2]. Positive levels of GAD65 antibodies were observed in 32/54 patients [fig.3]. Oral antihyperglycemia therapy with sulphonylureas was discontinued and insulin therapy initiated. The HbAIc levels were checked in 3 months after the start of insulin therapy. The positive changes in HbAIc parameters from the baseline were verified in all of them. Coexistence of autoimmune thyroiditis and type 2 diabetes took place in 28/71 cases [fig.4]. Coexistence of autoimmune thyroiditis and autoimmune subtype of type 2 diabetes was verified in 18/71 patients [fig.5].

Conclusion: coexistence of autoimmune insulitis and type 2 diabetes in adults is not rare variant and early detection of the subgroup of autoimmune diabetes among the patients with type 2 diabetes is necessary for the proper treatment protocol and earlier start of insulin therapy.

\section{References}

1. Hawa MI, Kolb H, Schloot N et al (2013) Adult-onset autoimmune diabetes in Europe is prevalent with a broad clinical phenotype: Action LADA 7. Diabetes care 36:908-913

2. Mollo A, Hernandez M, Marsal JR et al (2013) Latent autoimmune diabetes in adults is perched between type 1 and type 2: evidence from adults in one region of Spain. Diabetes/metabolism research and reviews 29:446-451

3. Laugesen E, Ostergaard JA, Leslie RD (2015) Latent autoimmune diabetes of the adult: current knowledge and uncertainty. Diabetic medicine 32:843-852

4. Xiang Y, Huang G, Zhu Yet al (2018) Identification of autoimmune type 1 diabetes and multiple organ-specific autoantibodies in adultonset non-insulin-requiring diabetes in China: a population-based multicentre nationwide survey. Diabetes Obes Metab 21:893-902
Fig.1. Enrolled patients

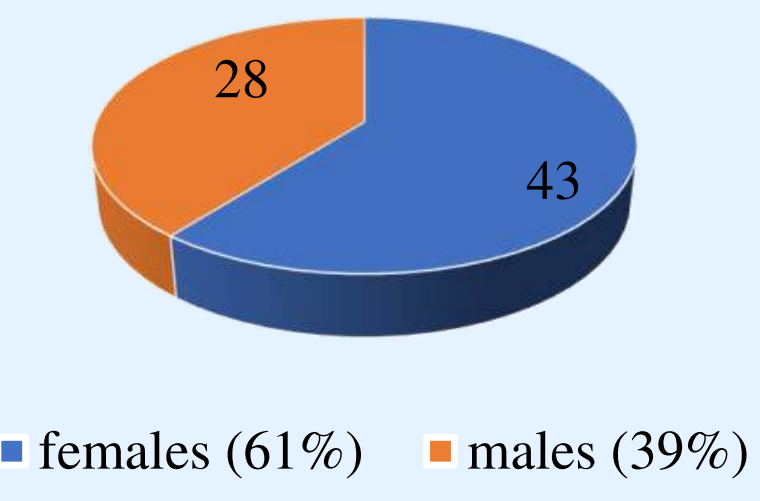

Age of 40-45 years and older

BMI varied from 32,2 to $38.5 \mathrm{~kg} / \mathrm{m} 2$

Results:

Glycated hemoglobin AIc (HbAIc) ranged from 9.5 to $13 \%$.

Fig.2. C-peptide serum levels

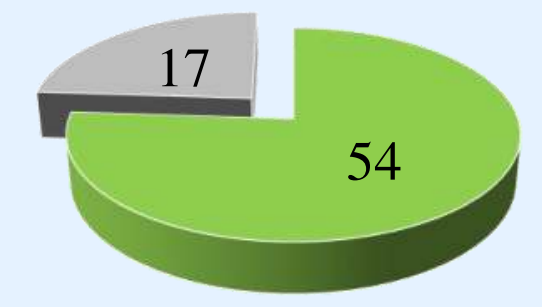

- low levels (76\%) $\square$ high levels $(24 \%)$

Fig.3. Levels of GAD65 antibodies

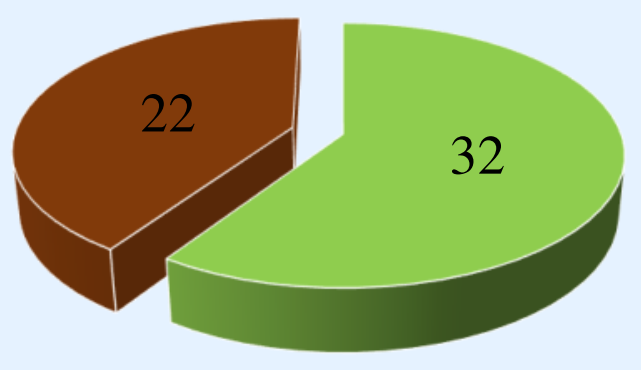

- Positive levels (59\%)

Fig.4. Coexistence of autoimmune thyroiditis and type 2 diabetes (39\%)

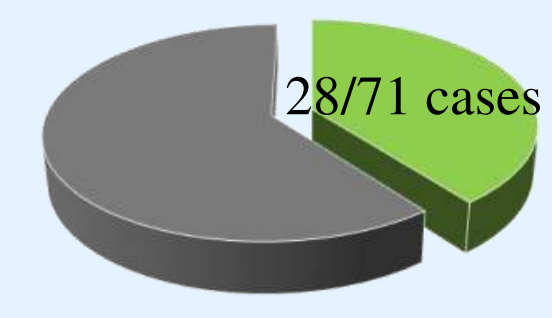

Fig.5. Coexistence of autoimmune thyroiditis and autoimmune subtype of type 2 diabetes (25\%)

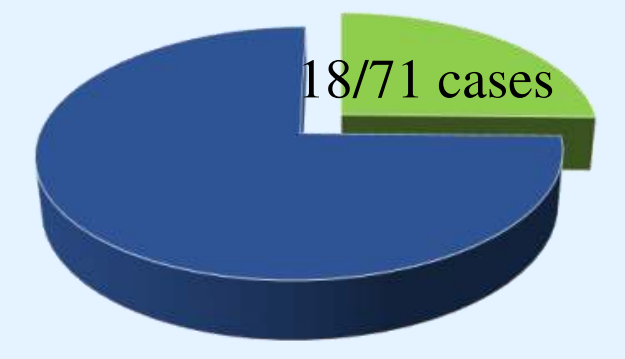

